\title{
Investigation of sources of gravity waves observed in the Brazilian equatorial region on 8 April 2005
}

\author{
Oluwakemi Dare-Idowu $^{1,2}$, Igo Paulino ${ }^{1}$, Cosme A. O. B. Figueiredo ${ }^{3}$, Amauri F. Medeiros ${ }^{1}$, Ricardo A. Buriti ${ }^{1}$, \\ Ana Roberta Paulino ${ }^{1,4}$, and Cristiano M. Wrasse ${ }^{3}$ \\ ${ }^{1}$ Unidade Acadêmica de Física, Universidade Federal de Campina Grande, Campina Grande, Brazil \\ ${ }^{2}$ Centre d'Etudes Spatiales de la BIOsphère, UMR 5126 CNES/CNRS/IRD, Université Paul-Sabatier, Toulouse, France \\ ${ }^{3}$ Divisão de Aeronomia, Instituto Nacional de Pesquisas Espaciais, São José dos Campos, Brazil \\ ${ }^{4}$ Departamento de Física, Universidade Estadual da Paraíba, Campina Grande, Brazil
}

Correspondence: Oluwakemi Dare-Idowu (oluwakemidareidowu@gmail.com)

Received: 4 June 2019 - Discussion started: 15 July 2019

Revised: 22 December 2019 - Accepted: 13 January 2020 - Published: 16 April 2020

\begin{abstract}
On 8 April 2005, strong gravity wave (GW) activity (over a period of more than $3 \mathrm{~h}$ ) was observed in São João do Cariri $\left(7.4^{\circ} \mathrm{S}, 36.5^{\circ} \mathrm{W}\right)$. These waves propagated to the southeast and presented different spectral characteristics (wavelength, period and phase speed). Using hydroxyl $(\mathrm{OH})$ airglow images, the characteristics of the observed GWs were calculated; the wavelengths ranged between 90 and $150 \mathrm{~km}$, the periods ranged from $\sim 26$ to $67 \mathrm{~min}$ and the phase speeds ranged from 32 to $71 \mathrm{~m} \mathrm{~s}^{-1}$. A reverse ray-tracing analysis was performed to search for the possible sources of the waves that were detected. The ray-tracing database was composed of temperature profiles from the Naval Research Laboratory Mass Spectrometer Incoherent Scatter (NRLMSISE-00) model and SABER measurements as well as wind profiles from the Horizontal Wind Model (HWM) and meteor radar data. According to the ray tracing result, the likely source of these observed gravity waves was the Intertropical Convergence Zone, which caused intense convective processes to take place in the northern part of the observatory. Also, the observed preferential propagation direction of the waves to the southeast could be explained using blocking diagrams, i.e. due to the wind filtering process.
\end{abstract}

\section{Introduction}

Since the publication of the pioneering works of Hines (1960) in the 1960s on the detection of irregular motions "gravity waves" (GWs) in the upper atmosphere, there have been numerous rapid advancements in this field of study. GWs are the result of disturbances that occur in atmospheric fluids and largely impact the upper mesosphere-thermosphere region (e.g. Fritts and Alexander, 2003). Potential sources of these waves are cold fronts (e.g. Plougonven et al., 2017), tropospheric convection (e.g. Vadas et al., 2009), wind shear (e.g. Clemesha and Batista, 2008), topography and wave breaking (e.g. Sarkar and Scotti, 2017), and solar eclipses (e.g. Marlton et al., 2016). These atmospheric structures have been identified as a key component in the transportation of energy in the mesosphere-lower thermosphere (MLT) region (e.g. Fritts and Luo, 1993; Medeiros et al., 2007; Campos et al., 2016).

Internal GWs are generated as adjustment radiations whenever a sudden change in forcing causes the atmosphere to depart from its large-scale balanced state. Such forcing anomaly occurs during a solar eclipse (Campos et al., 2016; Marlton et al., 2016). The intrinsic properties of the GWs (observed horizontal phase speed, propagation direction, observed period and horizontal wavelength) can be calculated directly from airglow images using spectral analysis. Utilising the dispersion relation, the vertical wavelength can also be computed (Vargas et al., 2009). GWs can be summarised as large-scale waves, medium-scale waves and small-scale waves. Small-scale GWs are characterised by horizontal wavelengths of tens of kilometres (Medeiros et al., 2003), medium-scale GW wavelengths range from $\sim 100$ to $400 \mathrm{~km}$, and large-scale waves have high phase speeds 
and travel larger horizontal distances than the small- and medium-scale waves (Vadas et al., 2009).

In the MLT region, there are several continuous chemical reactions such as OH airglow emissions (e.g. Sivjee, 1992; Taylor et al., 2009; Campos et al., 2016). These emissions, along with several others, have been used by many authors as a proxy for investigating GW activities. Airglow emissions are faint luminescence that is produced as a result of the emission of electromagnetic radiation by excited ionised or neutral atoms or molecules. These luminosities are usually captured by the all-sky imager (ASI) array (e.g. Wallace and Chamberlain, 1959; Krasovskij and Šefov, 1965).

To identify source location of GWs, the reverse ray-tracing method has been widely adopted. Several researchers have successfully implemented this technique to identify the generation points of these waves under different atmospheric conditions using airglow images (e.g. Hecht et al., 1994; Brown et al., 2004; Wrasse et al., 2006; Vadas et al., 2009; Pramitha et al., 2015; Sivakandan et al., 2016). Wrasse et al. (2006) carried out a comprehensive study of GWs observed over Brazil and Indonesia and concluded that most of the studied waves have sources in the troposphere. Similarly, Vadas et al. (2009) studied the propagation of GWs observed during the SpreadFEx campaign in Brazil and found that the likely sources of these waves were deep convection in Brazil. In addition, Pramitha et al. (2015) identified that $64 \%$ of the observed GWs over Gadanki, India, originated from the upper troposphere, while the remaining GWs were seen to have been ducted in the mesosphere. Sivakandan et al. (2016) also studied GWs observed in the southern part of India and associated the sources with convection.

The objective of the current study is to extensively study strong GW activity observed in São João do Cariri $\left(7.4^{\circ} \mathrm{S}\right.$, $36.5^{\circ} \mathrm{W}$ ) on 8 April 2005 . More than $3 \mathrm{~h}$ of GW activity was observed, and the waves propagated exclusively to the southeast. Observations of different $\mathrm{GW}$ parameters during this time period indicate that the sources of these waves must be large, as a wide spectrum of GWs was produced. An explanation for this uncommon pattern is presented in this work, stemming from the investigation of the combined effect of the location of the source and the wind filtering process.

\section{Instrumentation}

\subsection{The all-sky imager}

The GWs detected in this study were observed using the ASI installed at the observatory in São João do Cariri. The ASI is an optical instrument that provides monochromatic maps of aurora and atmospheric airglow emissions at different wavelengths. It has been designed to keep track of the spatial and temporal variations of $\mathrm{OH}$, OI $557.7 \mathrm{~nm}$ and $\mathrm{OI} 630.0 \mathrm{~nm}$ airglow emissions (e.g. Paulino et al., 2010).
However, the present study only utilised the $\mathrm{OH}$ airglow images captured by the ASI at an altitude of $87 \mathrm{~km}$. This instrument is comprised of a fish-eye (f/4) lens, a telecentric lens system, a field of view of $180^{\circ}$, a computer-controlled filter wheel with several slots for the observation of different emissions and a camera with a charge-coupled device (CCD) that is used as a photodetector to increase sensitivity. More technical and operational details about this particular imager at São João do Cariri can be found in previous works (Medeiros et al., 2007; Paulino et al., 2012).

\subsection{SABER/TIMED satellite}

The Sounding of the Atmosphere using Broadband Emission Radiometry (SABER) is one of the four instruments onboard the Thermosphere Ionosphere Mesosphere Energetics and Dynamics (TIMED) satellite. The vertical temperature measurements from 20 to $108 \mathrm{~km}$ were observed on 8 April 2005 over the São João do Cariri area $\left(7.4^{\circ} \mathrm{S}, 36.5^{\circ} \mathrm{W}\right)$ from this instrument (SABER). Data from the Naval Research Laboratory Mass Spectrometer Incoherent Scatter (NRLMSISE00) atmospheric model (Picone et al., 2002) were utilised to supplement the measurements at unavailable heights, namely 0 19 and $109-400 \mathrm{~km}$. These measurements were used to provide vertical profiles of kinetic temperature, pressure, geopotential height, and volume mixing ratios for the trace species (Mertens et al., 2001).

\subsection{The SKiYMET meteor radar}

The All-Sky Interferometric Meteor Radar (SKiYMET) system located at São João do Cariri $\left(7.4^{\circ} \mathrm{S}, 36.5^{\circ} \mathrm{W}\right)$ provided measurements of the horizontal wind speed and direction in the MLT $(81-99 \mathrm{~km})$. The radar is composed of Yagi antennas: five receiving antennas and one transmitting antenna that operate at $35.24 \mathrm{MHz}$ with a maximum power of $12 \mathrm{~kW}$. This instrument detects the trail left behind by vaporised meteors, determines the angle-of-arrival using the phase difference between the receiving antennas, and then measures the radial velocity using the derivation of the speed and direction of the atmospheric winds carrying the meteor trail at a specified altitude. The phase delay between the transmitted and received signal is used to determine the position of the trail. Further details about this radar are presented in previous works (e.g. Hocking et al., 2001; Egito et al., 2018; Paulino et al., 2015).

\section{Methodology and data analysis}

\subsection{Determination of GW parameters}

To obtain the characteristics of the detected GWs, a twodimensional fast Fourier transform (FFT) was used in specified batches of $\mathrm{OH}$ airglow images. The preprocessing of the airglow images can be summarised using the following procedures: 
Table 1. The observed properties of the five GWs.

\begin{tabular}{lccrccc}
\hline GWs & $\begin{array}{c}\text { Time } \\
(\mathrm{UTC})\end{array}$ & $\begin{array}{c}\tau \\
(\mathrm{min})\end{array}$ & $\begin{array}{r}\lambda_{\mathrm{H}} \\
(\mathrm{km})\end{array}$ & $\begin{array}{c}\text { Propagation } \\
\text { direction }\left({ }^{\circ}\right)\end{array}$ & $\begin{array}{c}\mathrm{cH} \\
\left(\mathrm{m} \mathrm{s}^{-1}\right)\end{array}$ & $\begin{array}{c}\mathrm{SD} \\
(\mathrm{km})\end{array}$ \\
\hline $\mathrm{GW}_{1}$ & $20: 22$ & 26.3 & 90.2 & 139.8 & 57.2 & \\
$\mathrm{GW}_{2}$ & $21: 33$ & 66.9 & 144.5 & 131.2 & 36.0 & \\
$\mathrm{GW}_{3}$ & $22: 56$ & 64.2 & 125.8 & 125.0 & 32.7 & 24.0 \\
$\mathrm{GW}_{4}$ & $23: 29$ & 40.8 & 142.0 & 142.0 & 58.0 & \\
$\mathrm{GW}_{5}$ & $23: 58$ & 35.0 & 149.2 & 150.9 & 71.0 & \\
\hline
\end{tabular}

- rotation of the image to fit the top of the image with the northern geographic region;

- removal of low-frequency waves by applying the Butterworth high-pass filter;

- contrast enhancement and unwarping of the airglow images for FFT analysis.

Figure 1a shows one of the raw $\mathrm{OH}$ images obtained from the São João do Cariri ASI at the Observatório de Luminescência Atmosférica de Paraiba (OLAP). This image is a typical example of the GWs observed during this night (8 April 2005). The ripples enclosed in the red box in Fig. 1a represent the GW structure. This particular GW was captured by the ASI at $\sim 23: 58$ UT on 8 April 2005. However, the image was contaminated by stars (bright circles), the Milky Way (white streak running from the bottom northwest in a southeasterly direction), tree branches/leaves (eastern edge of the image) and the tops of buildings (shown by the red arrow). Figure $1 \mathrm{~b}$ portrays a clearer image after image processing; Fig. 1c represents the unwarped version of the previous image; Fig. 1d shows the spectrum of the encapsulated GW. The bright red circle that depicts the amplitude of the GW and its positioning also provides the propagation direction of the wave. Table 1 presents a summary of all of the GWs observed during this night. Additional information about the cross-spectrum analysis used in the present study to obtain the characteristics of these waves can be found in Wrasse et al. (2006).

\subsection{The atmospheric profile}

The SABER instrument provided temperature measurements from 23:46 to 23:51 UT on 8 April and from 08:35 to 08:40 UT on 9 April for altitudes between 20 and $108 \mathrm{~km}$. Linear interpolation was then carried out between 23:51 (8 April) and 08:35 UT (9 April) for the same altitude range. In addition, numerical values for temperature were obtained from the NRLMSISE-00 model for time (12:00 to 22:00) on 8 April 2005 and for missing heights (0-19 and 109$400 \mathrm{~km}$ ) to supplement the SABER measurements. Finally, a 13-profile temperature dataset with a temporal resolution of $2 \mathrm{~h}$ was constructed for the whole period. However, some discrepancies were observed where points intersected; hence, the data were smoothened so that the model could seamlessly match the measurements at these junctions. This methodology was discussed by Paulino et al. (2012).

The SKiYMET radar provided the zonal and meridional wind measurements from 81 to $99 \mathrm{~km}$ every $3 \mathrm{~km}$ from 00:00 to 23:00 on 8 and 9 April 2005 at a temporal resolution of $1 \mathrm{~h}$. Similarly, numerical interpolation was used to provide the spatial resolution of the wind at a $2 \mathrm{~h}$ temporal resolution, as for the temperature profile, with supporting data from the Horizontal Wind Model (HWM) (Drob et al., 2008) from 0 to $80 \mathrm{~km}$, and above $100 \mathrm{~km}$.

From the temperature and wind measurements, other atmospheric parameters representing the atmospheric state of the study period were obtained. The pressure $P(z)=$ $P_{0} \exp ^{\left(-\int_{0}^{z} \frac{g}{R T} \mathrm{~d} z^{\prime}\right)}$ was obtained using a combination of the ideal gas law $(P=R \rho T)$ and the hydrostatic balance equation $\left(\frac{\mathrm{d} P}{\mathrm{~d} z}=-g p\right.$, where $\rho$ is the density obtained from the MSIS model); the molecular weight was estimated from $X_{\mathrm{MW}}=\frac{1}{2}\left(X_{\mathrm{MW} 0}-X_{\mathrm{MW} 1}\right)\left[1-\tanh \frac{s-a}{\Delta_{a}}\right]+X_{\mathrm{MW} 1}$, where $X_{\mathrm{MW} 0}, X_{\mathrm{MW} 1}, \Delta_{a}$ and $a$ are constants given by 28.90 , $16.0,4.20$, and 14.90 respectively, while $s=\ln \rho$ (Vadas et al., 2009). Additional details about the reverse ray-tracing parameters can be found in Vadas (2007) and Vadas et al. (2009). Briefly, $T$ is the temperature, $g$ is the acceleration due to gravity, $R=\frac{8314.5}{X_{\mathrm{MW}}}$ is the gas constant, $z$ is the altitude, and $P_{0}$ is the pressure when $z=0$.

The scale height $H=-\rho / \mathrm{d} \rho / \mathrm{d} z$ is obtained from the ratio of the density to the derivative with respect to the altitude; the potential temperature is $\theta=T\left(P_{0} / P\right)^{R / C_{\mathrm{p}}}$, where $C_{\mathrm{p}}$ is the specific heat capacity at constant pressure; the Brunt-Väisälä frequency is $N=\sqrt{\left(\frac{g}{\Theta} \frac{\mathrm{d} \Theta}{\mathrm{d} z}\right)}$. See Vadas (2007) for further details.

\subsection{The reverse ray-tracing technique}

Every GW is influenced by the atmospheric winds with a velocity $\boldsymbol{V}=\left(\mathrm{V}_{i}, \mathrm{~V}_{j}, \mathrm{~V}_{k}\right)$ according to Eqs. (1) and (2). Equation (1) describes the ray path, and the refraction of the wave vector along the ray path is explained by Eq. (2). The tracing model employed in the study is based on Lighthill (1978).

$\frac{\mathrm{d} k_{i}}{\mathrm{~d} t}=-k_{j} \frac{\partial \mathrm{V}_{j}}{\partial x_{i}}-\frac{\partial \omega_{\mathrm{Ir}}}{\partial x_{i}}$

and

$\frac{\mathrm{d} x_{i}}{\mathrm{~d} t}=\mathrm{V}_{i}+\frac{\partial \omega_{\mathrm{Ir}}}{\partial x_{i}}=\mathrm{V}_{i}+\boldsymbol{c}_{\mathrm{g} i}$,

where both $i$ and $j=1,2,3$, and the repeated indices imply a summation; $\mathrm{V}_{i}$ represents the zonal, meridional, and vertical wind components. The spatial position of the wave is represented by $\mathbf{x}, \boldsymbol{k}$ is the wave vector, $\omega_{\mathrm{Ir}}$ is the real part of the intrinsic frequency obtained using the dispersion relation in Eq. (3) of Pramitha et al. (2015) and $\boldsymbol{c}_{\mathrm{g}}$ is the group velocity.

Numerical integration of the six ordinary differential equations, Eqs. (1)-(2), was performed using a fourth-order 


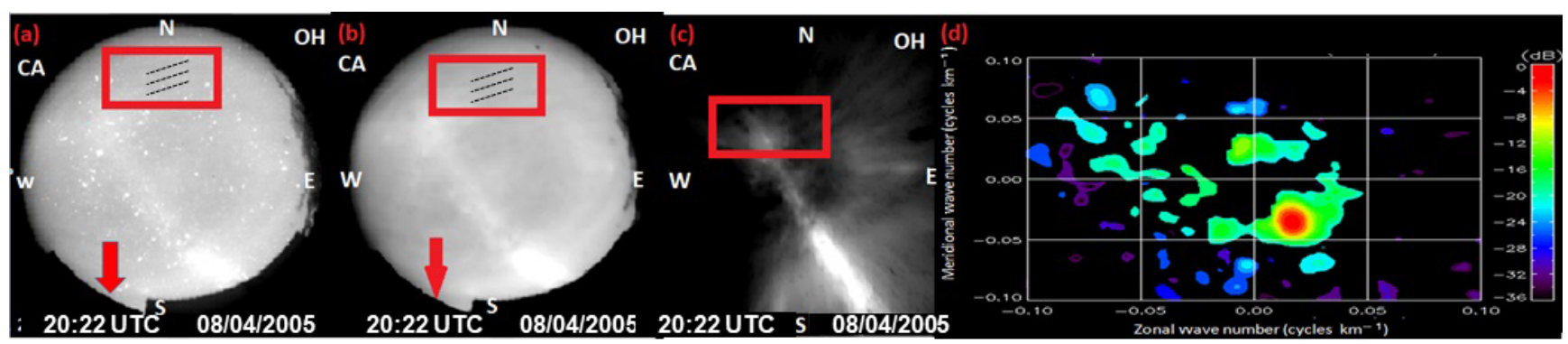

Figure 1. Illustration of the image processing. (a) Raw OH image collected from the ASI in São João do Cariri on 8 April 2005. For more details on the propagation of ripples, see the video clip in the Supplement. (b) The filtered image. (c) The unwarped and rotated image of the GW event inside the red box shown in the previous images for FFT analysis (d) The cross spectrum applied to the GW enclosed in the red box in (c).

Runge-Kutta scheme (Press, 2007). All of the observed and intrinsic properties of the GWs and the parameters representing the atmospheric condition were fed into the reverse raytracing (RRT) model. Four stopping conditions that were imposed constantly checked the propagation of the GWs into the background winds. When any of the conditions were violated, the ray-tracing integration was terminated. The main stopping criteria are as follows: (i) if the group velocity of the GW is close to the speed of sound $\left(\boldsymbol{c}_{\mathrm{g}} \geq 0.9 c_{\mathrm{s}}\right)$; (ii) if the real part of the intrinsic frequency tends to zero $\left(\omega_{\mathrm{Ir}} \rightarrow 0\right)$; (iii) if the vertical wavelength is greater than the viscosity length or the dissipation of the GWs; and (iv) if the momentum flow of the $\mathrm{GW} u_{\mathrm{GW}} w_{\mathrm{GW}}(z, t) \geq 10^{-5} u_{\mathrm{GW}} w_{\mathrm{GW}}$ at the height of $\mathrm{OH}$ airglow $(87 \mathrm{~km})$. Extensive details of the reverse ray-tracing algorithm employed in this study have been published in Paulino et al. (2012) and Pramitha et al. (2015).

\section{Results and discussion}

\subsection{Spectral analysis result}

The spectral results in Table 1 show that the horizontal wavelengths $\left(\lambda_{\mathrm{H}}\right)$ have a standard deviation of $24 \mathrm{~km}$ and a mean value of $130 \mathrm{~km}$ with large variability among the detected waves, while the propagating period $(\tau)$ of the waves ranged from 26 to $67 \mathrm{~min}$. These results agree with the reports of Medeiros et al. (2007) for waves detected at this observatory. Thus, it can be confidently concluded that the observed wavelengths are representative of the GWs at this observatory.

Figure 2 shows the travelling direction of the five GWs. From the polar chart, it is seen that all the waves are propagating southeast with an approximate azimuth of $\sim 134^{\circ}$ from the north. This is in favourable agreement with the results obtained from previous studies for the same observatory (Essien et al., 2018). This anisotropicity will be discussed later in this study.

Figure 3 shows the impact of the atmospheric wind on the period of the waves. It reveals the difference between the intrinsic period of the waves, which lacks atmospheric impact,

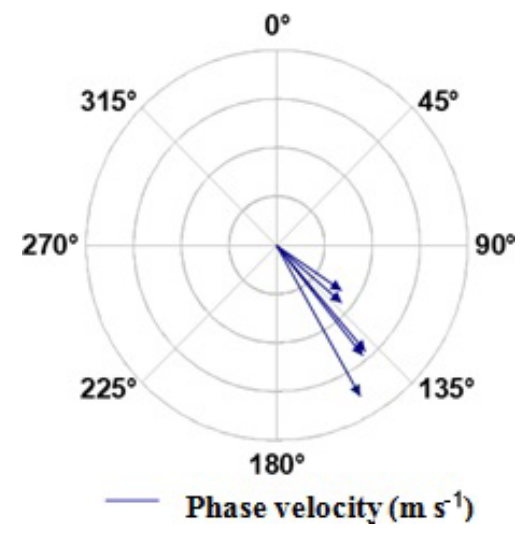

Figure 2. Compass graph showing wave velocities and the direction of propagation - each circle denotes a velocity of $20 \mathrm{~m} \mathrm{~s}^{-1}$.

and the observed period, which is susceptible to the influence of the atmospheric winds. However, it can be seen that the winds accelerated almost all of the GWs. The fluctuation in the period can be attributed to the variability of the horizontal winds. When the $\mathrm{GW}$ is travelling antiparallel to the wind direction, the second term on the left side of this equation $\omega_{\mathrm{I}}=\omega_{o}-k_{\mathrm{H}} V_{\mathrm{GW}}$ would become negative, consequently forcing the observed frequency to be smaller than the intrinsic frequency - this invariably results in an increase in the observed periods.

One important finding from the spectral analysis is that there is a wide spectrum of GWs being generated. This strongly suggests that the source of these GWs must be large (Vadas and Fritts, 2009). In order to investigate the likely sources of these waves, the next section of this work presents and discusses the results from the ray tracing.

\subsection{Reverse ray tracing (RRT)}

These five waves have different spectral characteristics, and they were all also observed at different times. Using the reverse ray-tracing technique to back trace these GWs from the point of observation in the airglow layer to their generation 


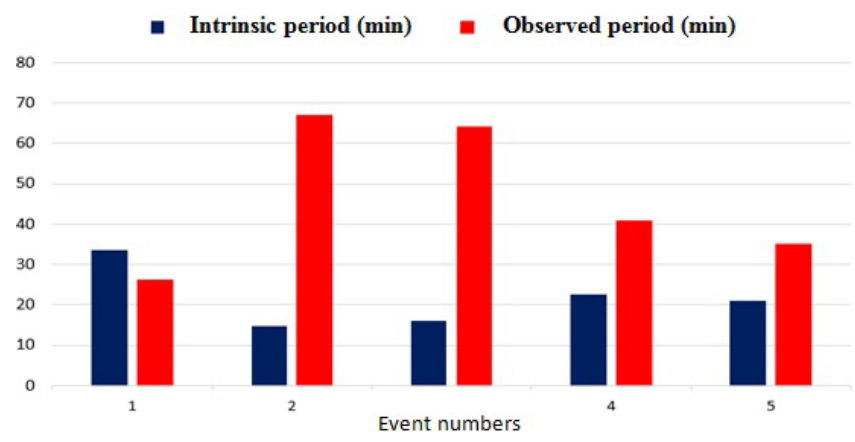

Figure 3. Bar chart of the intrinsic and observed periods.

source, we present the following results of the RRT analysis. During the backward tracing, we assumed that critical levels are not encountered, as explained in Sect. 3.3.

Panels (a) in Figs. 4-8 are the ray paths as a function of the altitude and time and describe the influence of various wind conditions on each GW event. The light cyan line indicates the trajectory of the GW under zero wind influence, whereas the black line shows the travel path of the wave under the influence of HWM wind. This provides a better understanding of the impact of atmospheric winds on the travel paths of GWs from the middle to the lower atmosphere. The cyan and black shaded circles represent the time of generation of these waves under zero and modelled wind scenarios respectively. The dashed cyan and solid black lines represent the trajectory of the waves under zero and modelled wind conditions respectively.

Panels (b) in Figs. 4-8, however, describe the wave path as a function of longitude-latitude, thereby providing a closer view of the exact generation point of these waves linearised over the map. The dark blue clouds represent the corresponding convective processes observed at the exact time that these GWs were detected. The black shaded triangle represents the precise location of the ASI at the OLAP.

Figure $4 \mathrm{~b}$ shows a GW propagating southeastward. Under the modelled wind conditions, this wave was generated at 17:00 UT of the same day, whereas under zero wind influence, it was triggered at 16:30 UT. Thus, the atmospheric winds were favourable and accelerated the travel speed. Figure $4 \mathrm{~b}$ zooms in on the actual source point of this wave. With a horizontal wavelength of $90 \mathrm{~km}$ and phase velocity of $57.2 \mathrm{~m} \mathrm{~s}^{-1}$, the reverse ray-tracing result points to the source being in the northern region of the detection point (OLAP). Even though the HWM-14 winds and zero winds are not equal, the GWs propagated through a similar path under both wind conditions. Below $70 \mathrm{~km}$, the GW propagates rapidly in the southeasterly direction, which could be due to the strong winds.

Similarly, the ray-tracing results show backward propagation of $\mathrm{GW}_{2}$ that was detected at 21:33 UT (Fig. 5a). This shows a similar analysis to $\mathrm{GW}_{1}$. However, $\mathrm{GW}_{2}$ suffered greater wind acceleration $\sim 12 \mathrm{~h}$, as did $\mathrm{GW}_{3} \sim 10 \mathrm{~h}$. More-
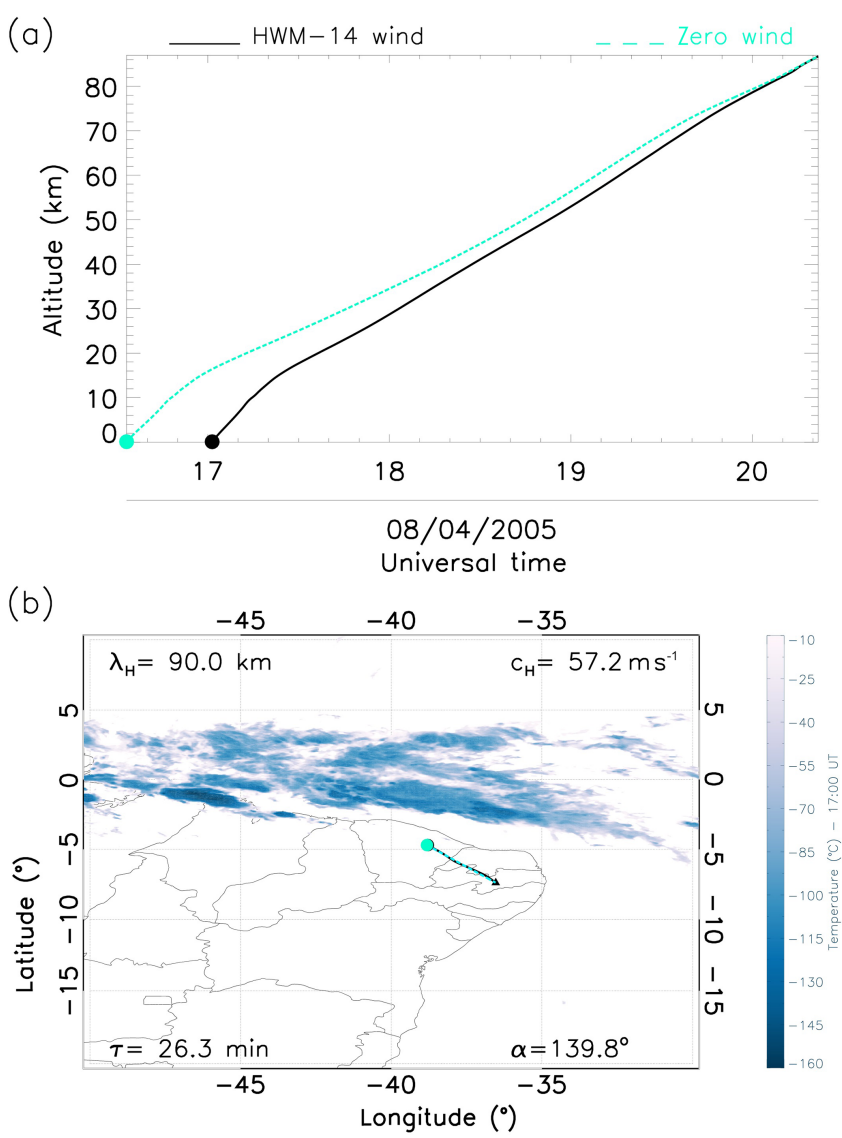

Figure 4. Reverse ray-tracing results for $\mathrm{GW}_{1}$. (a) Altitude as a function of time: dashed cyan and solid black lines show the ray paths for the zero winds and modelled winds respectively as well as the time cross section with zero wind and modelled winds. (b) Latitude-longitude cross section over convective cloud activities: black and cyan dots show the location of sources for zero winds and modelled winds respectively; the black triangle represents the exact location of the ASI, and the dark blue plumes represent convective activities in the region.

over, just as in $\mathrm{GW}_{1}$, both $\mathrm{GW}_{4}$ and $\mathrm{GW}_{5}$ were accelerated by the modelled winds by only $\sim 2$ and $1 \mathrm{~h}$ respectively.

It was observed that GWs 2, 3, 4 and 5, which had mean horizontal wavelengths of $140 \mathrm{~km}$, propagated more than $\sim 1300 \mathrm{~km}$ from their source region. All four of these GWs are believed to have originated from the same source due to the resemblance in their characteristics. In $\mathrm{GW}_{1}$, in comparison to the others, the bottom side of the convective sources (dark blue clouds) appears to be farther from the point that we established (traced) as the GW's source (black and cyan dots). However, this particular wave is still linked to the convective processes as its source. According to Vadas and Fritts (2009), the actual convective area is usually larger than the cloudy areas.

Figures $4 \mathrm{~b}-8 \mathrm{~b}$ showed the ray paths for the GWs, considering the normal and zero wind conditions. In the first panel 
(a)

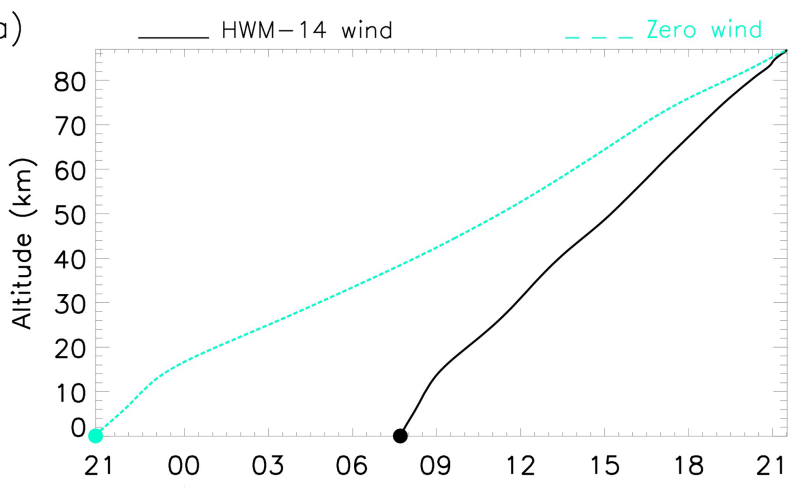

08/04/2005

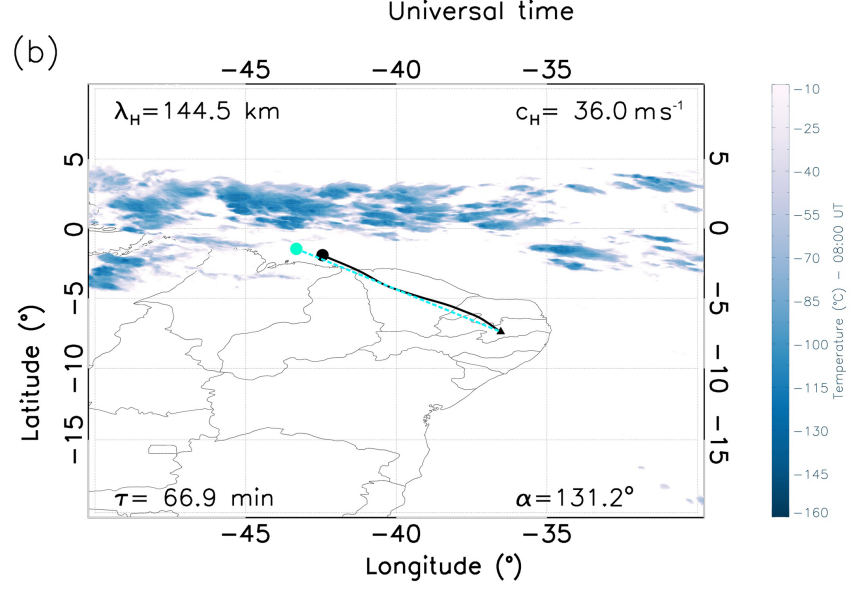

Figure 5. Same as in Fig. 4 but for $\mathrm{GW}_{2}$.

of $\mathrm{GW}_{3}$, the zero wind condition was applied, (cyan line) and the modelled wind condition (black line) was a horizontal wavelength of $90 \mathrm{~km}$, a phase velocity of $57.2 \mathrm{~m} \mathrm{~s}^{-1}$, a propagation angle of $139.8^{\circ}$, a $26.3 \mathrm{~min}$ period and a vertical wavelength of $90.2 \mathrm{~km}$. In Fig. 6a, it can be noticed that $\mathrm{GW}_{3}$ attained a height of $87 \mathrm{~km}$ for both wind conditions. A slight shift can be observed as there is a similarity between both conditions.

The five GWs were all traced back to convection activities occurring in the northern region of the OLAP observatory. These activities have been identified as possible generators of these waves. However, one question is evident: why do all of the waves propagate in a southeasterly direction when the Intertropical Convergence Zone (ITCZ) extends horizontally and covers the northern region of the observatory? These findings are in close agreement with previous results from different studies at this laboratory (e.g. Medeiros et al., 2003 and Essien et al., 2018). It is expected that the GWs should propagate in all directions in accordance with the location of the ITCZ. Thus, further investigation was carried out to explain this anisotropy. To better understand the physical mechanism that is producing the anisotropy, blocking diagrams have been used to investigate the role of the wind in the filtering process of these GWs.

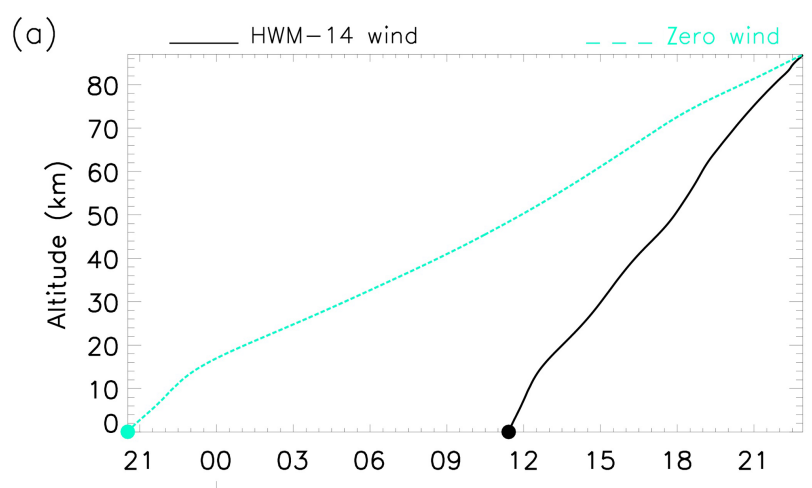

$08 / 04 / 2005$

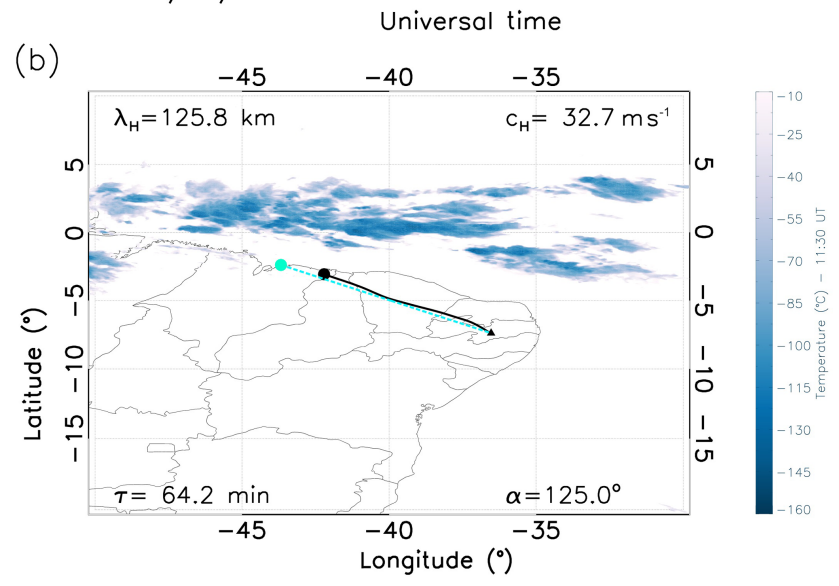

Figure 6. Same as Fig. 4 but for $\mathrm{GW}_{3}$.

Atmospheric winds are the default features of a real atmosphere. As these GWs propagate in the wind direction into the upper atmosphere, they become susceptible to the Doppler effect and critical level dissipation (Bretherton, 1966). The critical level marks the region where the horizontal wind component annuls the wave's horizontal phase speed (Medeiros et al., 2003). This region is very important, as it decides how and if a travelling wave would propagate further. To understand the anisotropy of these GWs, we apply the critical level theory of the atmospheric GW filtering (Fritts and Geller, 1976; Fritts, 1979). Based on the relation outlined by Gossard and Hooke (1975), the intrinsic frequency of the GW under the influence of both horizontal wind components can be described by Eq. (3) as follows:

$\omega_{\mathrm{I}}=\boldsymbol{k}(c-\boldsymbol{V})$,

where $\boldsymbol{k}$ is the magnitude of the horizontal wave vector, $\boldsymbol{V}$ represents the two horizontal wind components, and $c$ is the horizontal phase speeds of the GWs. Equation (4) can also be re-expressed in terms of the zonal and meridional components. Further details can be found in the works of Medeiros et al. (2003), Campos et al. (2016) and Paulino et al. (2018). According to $\mathrm{cH}=U \cos \varnothing+V \sin \varnothing$, where $\mathrm{cH}$ represents the phase speeds of these waves, $U$ is the zonal wind component and $V$ is the meridional wind component, we con- 

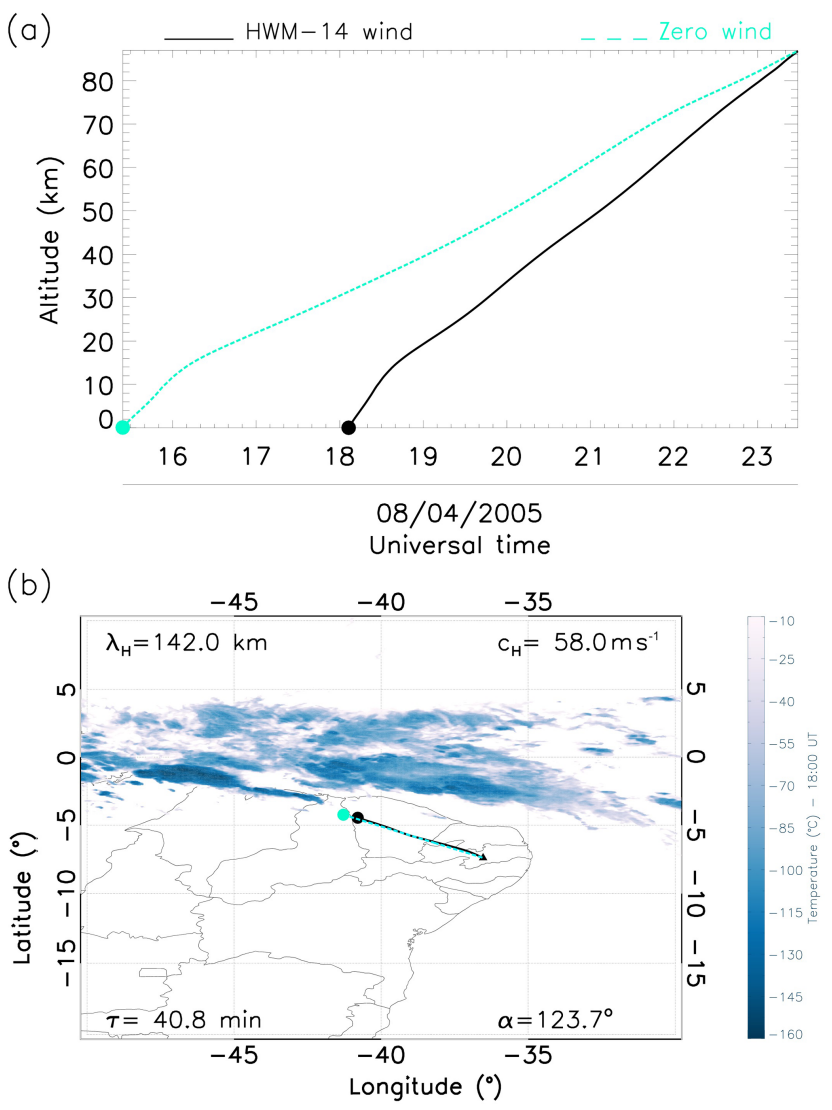

Figure 7. Same as Fig. 4 but for $\mathrm{GW}_{4}$.

structed blocking diagrams using the azimuthal angles $(\varnothing)$. With input winds from HWM, we aimed to understand the wind filtering effects on the GWs, to investigate why all of the waves had a preferential propagation direction, and also to detect regions where the phase speed of the GWs was less than or equal to the velocity of the winds. The results of the three-dimensional blocking diagrams are shown in Fig. 9.

Constructing a polar chart as a function of the azimuthal angles and phase speeds using the wind data from HWM and the SKiYMET radar, we show where the phase speeds of these GWs equaled the wind speed of the background. The critical levels were projected into the blocking diagram for each horizontal wind speed and azimuth of the corresponding GWs. If the phase speed of the GW is trapped in the blocking lines, it represents that the wave is prohibited from propagating upwards.

Figure 9 allows the detection of regions where $\omega_{\mathrm{I}} \leq 0$ on the night of 8 April 2005 for the $\mathrm{OH}$ emission layer. Every circle in this diagram shows the critical level of the vertical propagation of these waves. The red and black meshed areas signify the measured and modelled wind components respectively, and the green arrow represents the magnitude and direction of the detected GW. The theory of the filtering process of GWs disallows wave propagation into the
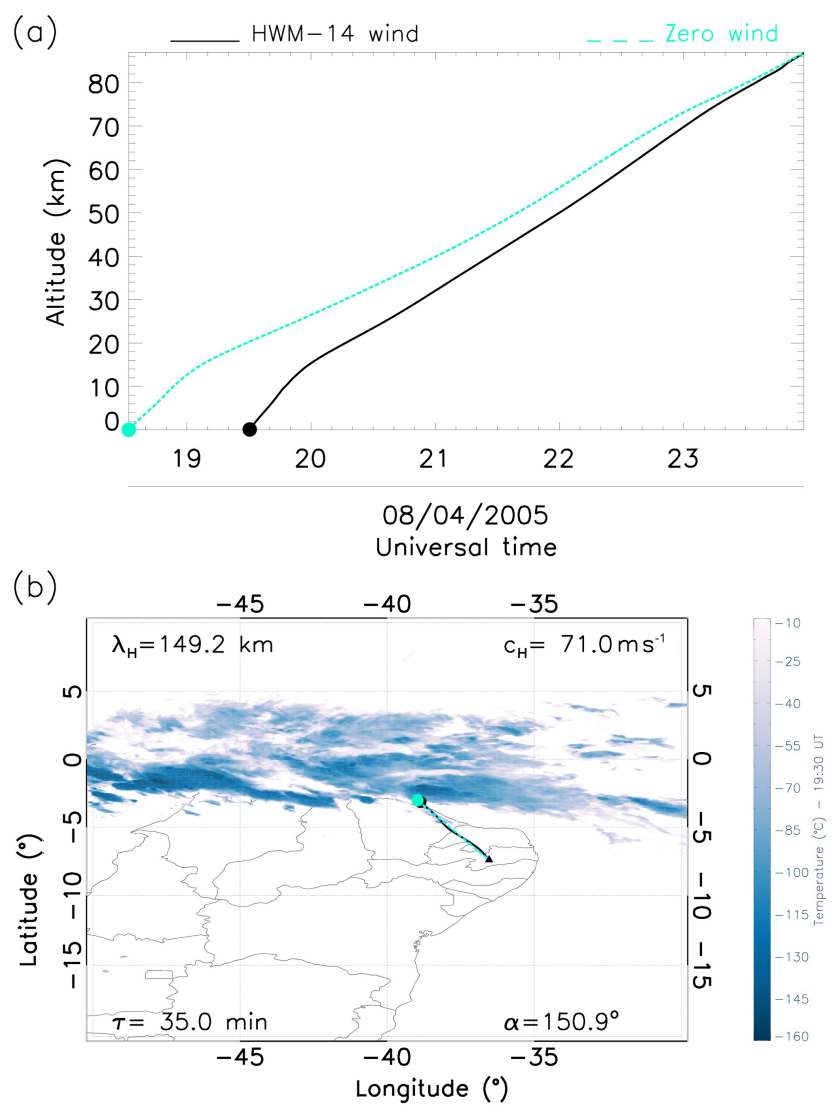

Figure 8. Same as Fig. 5 but for $\mathrm{GW}_{5}$.

shaded region due to the effect of the critical levels (Paulino et al., 2018).

Similarly, we observed that the phase velocity of all of the GW events was indeed greater in magnitude than the blocking area. These GWs had strong speed and momentum, which was sufficient to escape and easily propagate through the critical levels. It is important to note the main contributions to the blocking area were due to the measured wind in the mesosphere and lower thermosphere, making this analysis strongly confident.

Thus, these detected waves avoided and escaped absorption in the forbidden regions by travelling at these interesting angles. Moreover, the anisotropy of these waves means that the source location of the wave must in the northwest, as the location of this wave source played a key role in this preferential travelling (Fritts et al., 2008; Campos et al., 2016). The sources of these waves were identified as the convective processes in the ITCZ zone.

\section{Conclusions}

Using OH airglow images captured by the ASI at São João do Cariri, we investigated the sources of specific GWs observed on the night of 8 April 2005 in the OH airglow layer. Employ- 

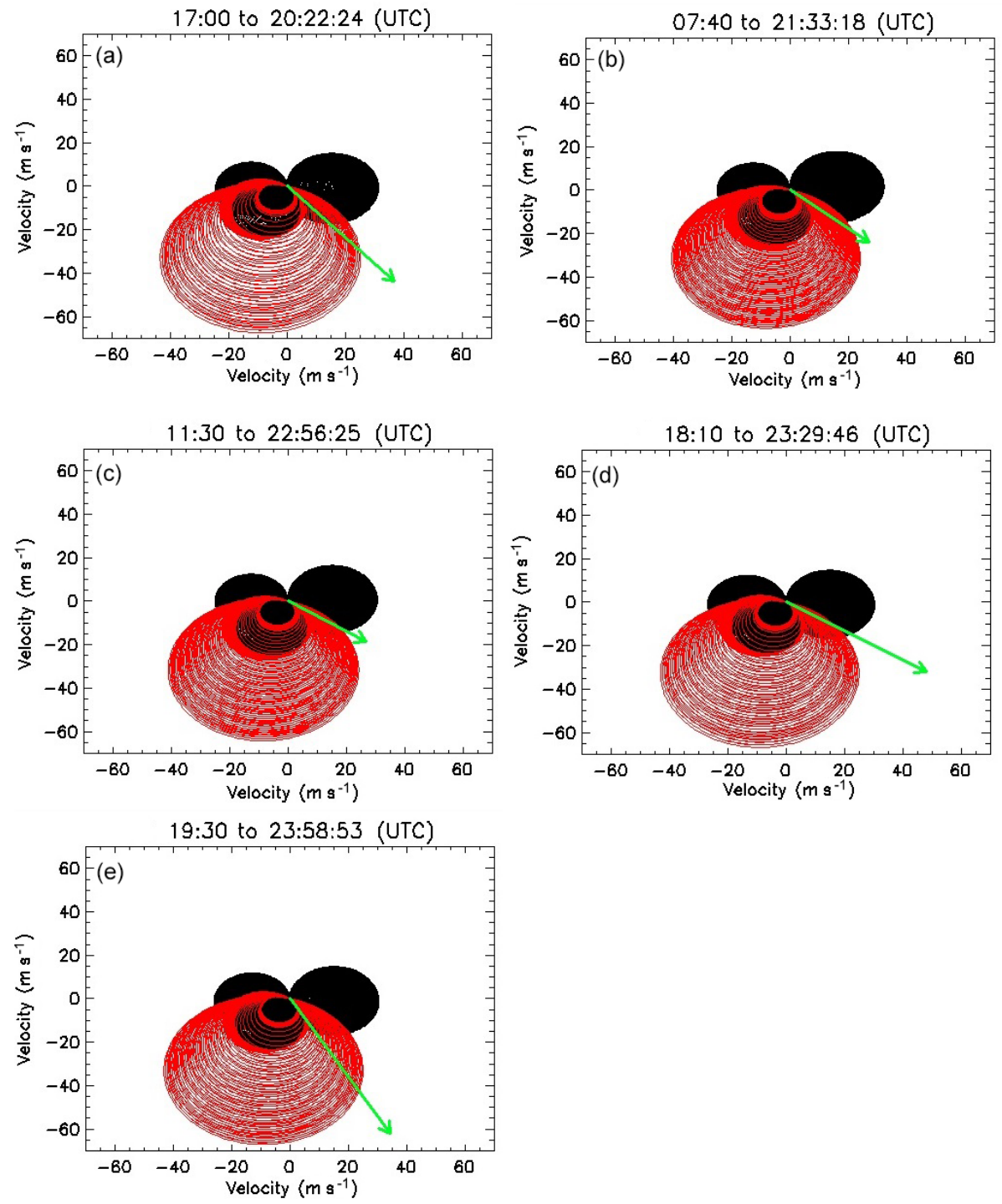

Figure 9. (a) Two-dimensional blocking diagrams for the $\mathrm{GW}_{1}$, (b) $\mathrm{GW}_{2}$, (c) $\mathrm{GW}_{3}$, (d) $\mathrm{GW}_{4}$, and (e) $\mathrm{GW}_{5}$ events observed in the $\mathrm{OH}$ layer $(87 \mathrm{~km})$. The green arrow depicts the magnitude and direction of the phase velocity of each $\mathrm{GW}$. The red and black meshed regions represent the respective magnitude and the direction of the restricted area for the propagation of the wave to the $(\mathrm{OH})$ layer.

ing a spectral analysis method, we obtained the characteristics of five major GW events with horizontal wavelengths concentrated between 90 and $149 \mathrm{~km}$. The phase speeds were distributed between 32 and $71 \mathrm{~m} \mathrm{~s}^{-1}$, and the observed periods extended from 26 to $67 \mathrm{~min}$. These waves presented spectral characteristics that were very compatible with waves previously observed at the same site. In addition, southeasterly propagation suggested possible sources in the northwest of the observatory.

Focusing on the possible sources of these waves, we traced the trajectory of each of these waves from the $\mathrm{OH}$ layer $(87 \mathrm{~km})$ back into the troposphere using the meteor radar wind data, the HWM model winds, and zero winds. We found that the RRT pinpointed the source as active convective pro- cesses (in the ITCZ) in the northwest of the laboratory, as shown in the back tracing results presented above. However, a long strip of the ITCZ extended into the northern part of the observatory which suggests the generation of other GWs that should have been observed in the south and southwest of the observatory. Thus, the construction of blocking diagrams showed that only the spectrum of waves with propagation to the southeast were able to propagate vertically to the altitudes of the $\mathrm{OH}$ layer. Therefore, the filtering effect of GWs was decisive in explaining the presence of observed waves propagating to the southeast. 
Data availability. All-sky image data used in the course of this study can be requested from the Aerolume (UFCG) or Lume (INPE) groups (igo.paulino@df.ufcg.edu.br).

Supplement. The supplement related to this article is available online at: https://doi.org/10.5194/angeo-38-507-2020-supplement.

Author contributions. ODI wrote the paper. IP revised the paper and supervised the research. CAOBF calculated the deep cloudy convection over the OLAP area. ARP provided wind measurements from the meteor radar and revised the paper. RAB and AFM revised the text. CMW provided the spectral analysis for the observed gravity waves.

Competing interests. The authors declare that they have no conflict of interest.

Special issue statement. This article is part of the special issue "7th Brazilian meeting on space geophysics and aeronomy". It is a result of the Brazilian meeting on Space Geophysics and Aeronomy, Santa Maria/RS, Brazil, 5-9 November 2018.

Acknowledgements. Oluwakemi Dare-Idowu sincerely thanks the Coodernação de Aperfeiçoamento de Pessoal de nível Superior (CAPES) for the scholarship during her master's programme at UFCG. Igo Paulino is grateful to the Conselho Nacional de Desenvolvimento Científico e Tecnológico (CNPq) for their financial support and to UFCG for their strong financial support during the presentation of this work at the 27th IUGG Scientific Assembly. Cosme A. O. B. Figueiredo would also like to acknowledge financial support from FAPESP. Ana Roberta Paulino is grateful to $\mathrm{CNPq}$ and CAPES for their financial support.

Financial support. This research has been supported by the Coodernação de Aperfeiçoamento de Pessoal de nível Superior (CAPES), the Conselho Nacional de Desenvolvimento Científico e Tecnológico (grant nos. 303511/2017-6, 460624/2014-8 and 307653/2017-0), and Fundação de Amparo à Pesquisa do Estado de São Paulo (grant no. 2018/09066-8).

Review statement. This paper was edited by Inez Batista and reviewed by two anonymous referees.

\section{References}

Bretherton, F. P.: The propagation of groups of internal gravity waves in a shear flow, Q. J. Roy. Meteor. Soc., 92, 466-480, https://doi.org/10.1002/qj.49709239403, 1966.

Brown, L. B., Gerrard, A. J., Meriwether, J. W., and Makela, J. J.: All-sky imaging observations of mesospheric fronts in OI
$557.7 \mathrm{~nm}$ and broadband $\mathrm{OH}$ airglow emissions: Analysis of frontal structure, atmospheric background conditions, and potential sourcing mechanisms, J. Geophys. Res., 109, D19104, https://doi.org/10.1029/2003JD004223, 2004.

Campos J. A. V., Paulino, I., Wrasse, C. M., Medeiros, A. F., Paulino, A. R., and Buriti, R. A.: Observations of small-scale gravity waves in the equatorial upper mesosphere, Revista Brasileira de Geofísica, 34, 469-477, https://doi.org/10.22564/rbgf.v34i4.876, 2016.

Clemesha, B. and Batista, P.: Gravity waves and wind-shear in the MLT at $23^{\circ} \mathrm{S}$, Adv. Space Res., 41, 1472-1477, https://doi.org/10.1016/j.asr.2007.03.085, 2008.

Drob, D. P., Emmert, J. T., Crowley, G., Picone, J. M., Shepherd, G. G., Skinner, W., Hays, P., Niciejewski, R. J., Larsen, M., She, C. Y., Meriwether, J. W., Hernandez, G., Jarvis, M J., Sipler, D. P., Tepley, C. A., O’Brien, M. S., Bowman, J. R., Wu, Q., Murayama, Y., Kawamura, S., Reid, I. M., and Vincent, R. A.: An empirical model of the Earth's horizontal wind fields: HWM07, J. Geophys. Res., 113, A12304, https://doi.org/10.1029/2008JA013668, 2008.

Egito, F., Buriti, R. A., Fragoso Medeiros, A., and Takahashi, H.: Ultrafast Kelvin waves in the MLT airglow and wind, and their interaction with the atmospheric tides, Ann. Geophys., 36, 231241, https://doi.org/10.5194/angeo-36-231-2018, 2018

Essien, P., Paulino, I., Wrasse, C. M., Campos, J. A. V., Paulino, A. R., Medeiros, A. F., Buriti, R. A., Takahashi, H., AgyeiYeboah, E., and Lins, A. N.: Seasonal characteristics of smalland medium-scale gravity waves in the mesosphere and lower thermosphere over the Brazilian equatorial region, Ann. Geophys., 36, 899-914, https://doi.org/10.5194/angeo-36-899-2018, 2018.

Fritts, D. C.: The excitation of radiating waves and KelvinHelmholtz instabilities by the gravity wave-critical level interaction, J. Atmos. Sci., 36, 12-23, https://doi.org/10.1175/15200469(1979)036<0012:TEORWA>2.0.CO;2, 1979.

Fritts, D. C. and Alexander, M. J.: Gravity wave dynamics and effects in the middle atmosphere, Rev. Geophys., 41, 1003, https://doi.org/10.1029/2001RG000106, 2003.

Fritts, D. C. and Geller, M. A.: Viscous stabilization of gravity wave critical level flows, J. Atmos. Sci., 33, 2276-2284, https://doi.org/10.1175/15200469(1976)033<2276:VSOGWC>2.0.CO;2, 1976.

Fritts, D. C. and Luo, Z.: Gravity wave forcing in the middle atmosphere due to reduced ozone heating during a solar eclipse, J. Geophys. Res., 98, 3011-3021, https://doi.org/10.1029/92JD02391, 1993.

Fritts, D. C., Vadas, S. L., Riggin, D. M., Abdu, M. A., Batista, I. S., Takahashi, H., Medeiros, A., Kamalabadi, F., Liu, H.-L., Fejer, B. G., and Taylor, M. J.: Gravity wave and tidal influences on equatorial spread $\mathrm{F}$ based on observations during the Spread F Experiment (SpreadFEx), Ann. Geophys., 26, 32353252, https://doi.org/10.5194/angeo-26-3235-2008, 2008

Gossard, E. and Hooke, W.: Waves in the atmosphere: atmospheric infrasound and gravity waves- their generation and propagation, Elsevier Scientific Publishing Co., Amsterdam, 1975.

Hecht, J. H., Walterscheid, R. L., and Ross, M. N.: First measurements of the two-dimensional horizontal wave number spectrum from CCD images of the nightglow, J. Geophys. Res., 99, 11449 11460, https://doi.org/10.1029/94JA00584, 1994. 
Hines, C. O.: Internal atmospheric gravity waves at ionospheric heights, Can. J. Phys., 38, 1441-1481, https://doi.org/10.1139/p60-150, 1960.

Hocking, W. K., Fuller, B., and Vandepeer, B.: Real-time determination of meteor-related parameters utilizing modern digital technology, J. Atmos. Sol.-Terr. Phys., 63, 155-169, https://doi.org/10.1016/S1364-6826(00)00138-3, 2001.

Krasovskij, V. I. and Šefov, N. N.: Airglow, Space Sci. Rev., 4, 176198, https://doi.org/10.1007/BF00173881, 1965.

Lighthill, J.: Waves in Fluids, Cambridge Univ. Press, New York, 1978.

Marlton, G. J., Williams, P. D., and Nicoll, K. A.: On the detection and attribution of gravity waves generated by the 20 March 2015 solar eclipse, Philos. T. Roy. Soc. A, 374, 20150222, https://doi.org/10.1098/rsta.2015.0222, 2016.

Medeiros, A. F., Taylor M. J., Takahashi, H., Batista, P. P., and Gobbi, D.: An investigation of gravity wave activity in the low-latitude upper mesosphere: Propagation direction and wind filtering, J. Geophys. Res., 108, 4411, https://doi.org/10.1029/2002JD002593, 2003.

Medeiros, A. F., Takahashi, H., Buriti, R. A., Fechine, J., Wrasse, C. M., and Gobbi, D.: MLT gravity wave climatology in the South America equatorial region observed by airglow imager, Ann. Geophys., 25, 399-406, https://doi.org/10.5194/angeo-25399-2007, 2007.

Mertens, C. J., Mlynczak, M. G., López-Puertas, M., Wintersteiner, P. P., Picard, R. H., Winick, J. R., Gordley, L. L., and Russell III, J. M.: Retrieval of mesospheric and lower thermospheric kinetic temperature from measurements of $\mathrm{CO}_{2} 15 \mu \mathrm{m}$ Earth Limb Emission under non-LTE conditions, Geophys. Res. Lett., 28, 1391-1394, https://doi.org/10.1029/2000GL012189, 2001.

Paulino, A. R., Batista, P. P., Lima, L. M., Clemesha, B. R., Buriti, R. A., and Schuch, N.: The lunar tides in the mesosphere and lower thermosphere over Brazilian sector, J. Atmos. Sol.-Terr. Phys., 133, 129-138, https://doi.org/10.1016/j.jastp.2015.08.011, 2015.

Paulino, I., Medeiros, A. F., Buriti, R. A., Sobral, J. H. A., Takahashi, H., and Gobbi, D.: Optical observations of plasma bubble westward drifts over Brazilian tropical region, J. Atmos. Sol.-Terr. Phys., 72, 521-527, https://doi.org/10.1016/j.jastp.2010.01.015, 2010.

Paulino, I., Takahashi, H., Vadas, S., Wrasse, C., Sobral, J., Medeiros, A., Buriti, R., and Gobbi, D.: Forward raytracing for medium-scale gravity waves observed during the COPEX, J. Atmos. Sol--Terr. Phys., 90-91, 117-123, https://doi.org/10.1016/j.jastp.2012.08.006, 2012.

Paulino, I., Moraes, J. F., Maranhão, G. L., Wrasse, C. M., Buriti, R. A., Medeiros, A. F., Paulino, A. R., Takahashi, H., Makela, J. J., Meriwether, J. W., and Campos, J. A. V.: Intrinsic parameters of periodic waves observed in the OI6300 airglow layer over the Brazilian equatorial region, Ann. Geophys., 36, 265273, https://doi.org/10.5194/angeo-36-265-2018, 2018.

Picone, J. M., Hedin, A. E., Drob, D. P., and Aikin, A. C.: NRLMSISE-00 empirical model of the atmosphere: Statistical comparisons and scientific issues, J. Geophys. Res., 107, 1468, https://doi.org/10.1029/2002JA009430, 2002.
Plougonven, R., Jewtoukoff, V., Cámara, A., Lott, F., and Hertzog, A.: On the Relation between Gravity Waves and Wind Speed in the Lower Stratosphere over the Southern Ocean, J. Atmos. Sci., 74, 1075-1093, https://doi.org/10.1175/JAS-D-16-0096.1, 2017.

Pramitha, M., Venkat Ratnam, M., Taori, A., Krishna Murthy, B. V., Pallamraju, D., and Vijaya Bhaskar Rao, S.: Evidence for tropospheric wind shear excitation of high-phase-speed gravity waves reaching the mesosphere using the ray-tracing technique, Atmos. Chem. Phys., 15, 2709-2721, https://doi.org/10.5194/acp15-2709-2015, 2015.

Press, W. H., Brian, P. F., Saul, A. T., and William, T. V.: Numerical recipes: The Art of scientific computing, 3rd edn., Cambridge University Press, New York, 2007.

Sarkar, S. and Scotti, A.: Topographic Internal Gravity Waves to Turbulence, Annu. Rev. Fluid Mech., 49, 195-220, https://doi.org/10.1146/annurev-fluid-010816-060013, 2017.

Sivakandan, M., Paulino, I., Taori, A., and Niranjan, K.: Mesospheric gravity wave characteristics and identification of their sources around spring equinox over Indian low latitudes, Atmos. Meas. Tech., 9, 93-102, https://doi.org/10.5194/amt-9-93-2016, 2016.

Sivjee G. G.: Airglow hydroxyl emissions, Planet. Space Sci., 40, 235-242, https://doi.org/10.1016/0032-0633(92)90061-R, 1992.

Taylor, M. J., Pautet, P.-D., Medeiros, A. F., Buriti, R., Fechine, J., Fritts, D. C., Vadas, S. L., Takahashi, H., and São Sabbas, F. T.: Characteristics of mesospheric gravity waves near the magnetic equator, Brazil, during the SpreadFEx campaign, Ann. Geophys., 27, 461-472, https://doi.org/10.5194/angeo-27-461-2009, 2009

Vadas, S. L. and Fritts, D. C.: Reconstruction of the gravity wave field from convective plumes via ray tracing, Ann. Geophys., 27, 147-177, https://doi.org/10.5194/angeo-27-147-2009, 2009.

Vadas, S. L.: Horizontal and vertical propagation and dissipation of gravity waves in the thermosphere from lower atmospheric and thermospheric sources, J. Geophys. Res., 112, A06305, https://doi.org/10.1029/2006JA011845, 2007.

Vadas, S. L., Taylor, M. J., Pautet, P.-D., Stamus, P. A., Fritts, D. C., Liu, H.-L., São Sabbas, F. T., Rampinelli, V. T., Batista, P., and Takahashi, H.: Convection: the likely source of the medium-scale gravity waves observed in the $\mathrm{OH}$ airglow layer near Brasilia, Brazil, during the SpreadFEx campaign, Ann. Geophys., 27, 231-259, https://doi.org/10.5194/angeo-27-231-2009, 2009.

Vargas, F., Gobbi, D., Takahashi, H., and Lima, L. M.: Gravity wave amplitudes and momentum fluxes inferred from $\mathrm{OH}$ airglow intensities and meteor radar winds during SpreadFEx, Ann. Geophys., 27, 2361-2369, https://doi.org/10.5194/angeo27-2361-2009, 2009

Wallace, L. and Chamberlain, J. W.: Excitation of $\mathrm{O}_{2}$ atmospheric bands in the aurora, Planet. Space Sci., 2, 60-70, https://doi.org/10.1016/0032-0633(59)90060-1, 1959.

Wrasse, C. M., Nakamura, T., Takahashi, H., Medeiros, A. F., Taylor, M. J., Gobbi, D., Denardini, C. M., Fechine, J., Buriti, R. A., Salatun, A., Suratno, Achmad, E., and Admiranto, A. G.: Mesospheric gravity waves observed near equatorial and low-middle latitude stations: wave characteristics and reverse ray tracing results, Ann. Geophys., 24, 3229-3240, https://doi.org/10.5194/angeo-24-3229-2006, 2006. 